\title{
Detecting multi-atomic composite states in optical lattices
}

\author{
Anatoly Kuklov ${ }^{1}$ and Henning Moritz ${ }^{2}$ \\ ${ }^{1}$ Department of Physics, CSI, CUNY - Staten Island, New York, NY 10314 \\ ${ }^{2}$ Institute of Quantum Electronics, ETH Zurich, 8093 Zurich, Switzerland
}

\begin{abstract}
We propose and discuss methods for detecting quasi-molecular complexes which are expected to form in strongly interacting optical lattice systems. Particular emphasis is placed on the detection of composite fermions forming in Bose-Fermi mixtures. We argue that, as an indirect indication of the composite fermions and a generic consequence of strong interactions, periodic correlations must appear in the atom shot noise of bosonic absorption images, similar to the bosonic Mott insulator [S. Fölling, et al., Nature 434, 481 (2005)]. The composites can also be detected directly and their quasi-momentum distribution measured. This method - an extension of the technique of noise correlation interferometry [E. Altman et al., Phys. Rev. A 79, 013603 (2004)] - relies on measuring higher order correlations between the bosonic and fermionic shot noise in the absorption images. However, it fails for complexes consisting of more than three atoms.
\end{abstract}

\section{INTRODUCTION}

Atomic mixtures in optical lattices represent a novel laboratory system for the study of ultracold matter. They are intrinsically clean and feature a unique amount of tunability. Therefore they offer outstanding opportunities to investigate fundamental many-body systems exhibiting some very rich physics. A fascinating example is the formation of composite states, which are induced by the lattice and the interaction. Such states have recently been detected experimentally [1, 2, 3, 4].

Two-component mixtures of atoms of, say, sorts A and $\mathrm{B}$ provide the simplest example of such objects which can be viewed as diatomic AB quasi-molecules occurring if the A-B interaction is strong enough. It is interesting to note that the formation of composites is possible not only for attractive [5, 6] but also for repulsive interaction 7]. In the last case the pairing occurs between an atom of sort, say, A and a hole $\overline{\mathrm{B}}$ of the sort B. Moreover, multi-atomic complexes of the type $\mathrm{AB}_{n}$ with $n>1$ can form as well [8, 9, 10]. Even in strongly non-equilibrium situations quasi-bound atomic states can exist if the repulsive energy greatly exceeds a typical single particle bandwidth. This mechanism was first introduced for the point-like defects in solid He4 11] and realized recently in the optical lattice [12].

In this paper particular emphasis will be put on generic schemes for detecting the composites in the lattice BoseFermi mixtures since such mixtures have recently been studied experimentally [13, 14]. We will be referring to the composites of the type $\mathrm{FB}_{n}$ or $\mathrm{FB}_{n}, n=1,2,3, \ldots$ where $\mathrm{F}$ stands for a fermion and $\mathrm{B}(\overline{\mathrm{B}})$ denotes a boson (bosonic hole). However, all the conclusions can be easily extended to any other type of a composite.

The quasi-molecular complexes are induced by the lattice and consequently vanish in most cases once the lattice potential is removed. Since the standard method of detection in ultracold gases, namely time of flight imaging, entails the elimination of all trapping potentials, the detection of composites with this technique is challenging. In the example of Bose-Fermi mixtures, the forma- tion of such complexes - composite fermions - would lead to the destruction of the Fermi surface of the original fermions. Therefore the disappearance of the corresponding feature should be observable in the absorption image. However, this disappearance would by no means constitute a definite proof of the formation of composites since a variety of phenomena, e.g. heating, could also lead to the same observation.

In this letter we propose a method how such composites can be detected directly. The method is similar to the Hanbury Brown and Twiss noise correlation interferometry 15] applied to thermal sources of cold atoms 16. and proposed in ref. 17] for revealing non-trivial manybody states in ultracold atoms. Such a method has been successfully implemented in ref. [18, 19, 20] for bosonic and in ref. 21, 22] for fermionic systems.

Noise correlation spectroscopy relies on the fact that, while the momentum distribution itself shows no particular features, the atomic shot noise is correlated. In order to introduce the extended noise correlation method with a simple example, consider a model system with a single composite (quasi-molecule) consisting of one boson and one fermion. The quasi-momentum of the center of mass of the composite is denoted by $\mathbf{Q}$. When the lattice is switched off, the pair breaks and the fermionic and bosonic wavepackets expand independently from each other. The fermionic or the bosonic momenta distributions $n_{F}\left(\mathbf{q}_{1}\right)$ and $n_{B}\left(\mathbf{q}_{2}\right)$, respectively, by themselves, which can be measured after expansion, do not reveal any low energy structure in their individual momenta $\mathbf{q}_{1}, \mathbf{q}_{2}$ - they feature almost uniform backgrounds because binding of both atoms entails a wide range of their relative momenta. However, momentum conservation requires the momenta to add up to $\mathbf{Q}$. Therefore, if in an individual measurement, the fermion is found to have $\mathbf{q}_{\mathbf{1}}$, the boson must have $\mathbf{q}_{\mathbf{2}}=\mathbf{Q}-\mathbf{q}_{\mathbf{1}}$ and the correlation between the two enables one to measure the quasimomentum of the composite.

In the more general case with more than one pair in the system, the correlations between the bosonic and fermionic momentum distributions, which allow one to determine the quasi-momenta of the pairs, will only be 
observable in the noise $\delta n_{B, F}=n_{B, F}-\left\langle n_{B, F}\right\rangle$ of the measured momentum distributions. This is due to the fact that, besides the correlated pair events, there are also events due to the components originating from different pairs. The key point is that the momentum distribution of the (centers of mass of the) FB composites is reproduced by the noise correlator in the far field of free expansion

$$
I_{C F}(\mathbf{Q})=\int d \mathbf{X}_{2}\left\langle\delta n_{F}\left(\mathbf{q}_{1}\right) \delta n_{B}\left(\mathbf{q}_{2}\right)\right\rangle
$$

with $\mathbf{Q}=\mathbf{q}_{1}+\mathbf{q}_{2}$ fixed, and $\mathbf{q}_{1}=m_{F} \mathbf{X}_{1} / t=\mathbf{Q}-\mathbf{q}_{2}$, $\mathbf{q}_{2}=m_{B} \mathbf{X}_{2} / t$ being the dependent and independent variables, respectively. Here $m_{F}, m_{B}$ are the masses of fermionic and bosonic atoms, respectively, and $\mathbf{X}_{1}\left(\mathbf{X}_{2}\right)$ denotes the position in the far field where a fermion (boson) is detected. We point out that (11) is essentially the Fermi distribution function $\tilde{\rho}_{C F}(\mathbf{Q})$ of the composite fermions $\left(\tilde{\rho}_{C F}(\mathbf{Q}) \approx 1\right.$ for $|\mathbf{Q}| \leq Q_{C F}$ and $\tilde{\rho}_{C F}(\mathbf{Q}) \approx 0$ for $|\mathbf{Q}| \geq Q_{C F}$, where $Q_{C F}$ stands for the Fermi surface wavevector of the composites). We also show that in the repulsive Bose-Fermi mixture, the role of the center of mass momentum $\mathbf{Q}$ is played by $\mathbf{Q}_{-}=\mathbf{q}_{1}-\mathbf{q}_{2}$ similar to the case of the repulsive two-component bosonic mixture [17]. These properties of the correlator (11) will be discussed in detail in the appendix $\mathrm{A}$ and extended to a general case of $\mathrm{FB}_{n}$ and $\mathrm{FB}_{n}$ complexes in section $\amalg \mathrm{C}$

Detection of the Fermi edge in $I_{C F}(\mathbf{Q})$ may require quite low temperatures $T$ determined by the Fermi energy $E_{C F}$ of the composites. However, since a typical binding energy $E_{b}$ of the composites can be much larger than $E_{C F}$, a signature of the composites will be seen as long as $T<E_{b}$ (for $T>E_{b}$, the correlator (11) is essentially zero). Indeed, the very fact that $I_{C F}(\mathbf{Q})$ is finite and proportional to a typical density of the original fermions or bosons is a direct indication of the presence of the composites. Accordingly, as $T$ is lowered below $E_{C F}$, the formation of the Fermi edge in $I_{C F}(\mathbf{Q})$ will be observed.

In principle, detection of multi-atomic complexes quasi-molecules with the number of the atomic constituents exceeding two - can be done by extending the noise-correlator method, as explained in SecIC However, as will be discussed in SecIID such an extension to higher order correlators can lead to a dramatic increase of uncorrelated noise. For this case, we propose an indirect method to observe the complexes (Sec III). In this method, the presence of complexes of the type $\mathrm{FB}_{n}$ is revealed by imaging the density-density noise correlations of the original bosons, if their number is incommensurate with the number of the lattice sites. Then the resulting image is similar to the one observed in a bosonic Mott insulator [18, 19]. Such a feature (at zero temperature) turns out to be a consequence of a strongly interacting many-body ground state in which individual momenta of bosons are poorly defined and, therefore, strongly fluctuate.

\section{DESCRIPTION AND DIRECT DETECTION OF COMPOSITES IN OPTICAL LATTICES}

\section{A. The nature of the composite pairs}

Here we will give a brief account of the nature of equilibrium quasi-molecular states. In general, quasimolecular (composite) states form when the binding energy $E_{b}$ exceeds the temperature $T$ as well as the gain in kinetic energy the constituents would encounter by delocalizing from each other. For attractive interactions between bosons and fermions, composites states of the type FB are formed. For repulsive interaction, on the other hand, the quasi-molecules represent bound states of one fermion $\mathrm{F}$ and a bosonic hole $\overline{\mathrm{B}}[\mathbf{7}]$ or, similarly, one fermionic hole $\overline{\mathrm{F}}$ and a boson B. Binding of one fermion with more than one boson or bosonic hole is also possible if the number of bosons per site is large enough and the magnitude of the Bose-Fermi interaction $\left|U_{B F}\right|$ substantially exceeds the repulsion between bosons $U_{B B}[10]$. In this event the composites $\mathrm{FB}_{n}$ (for $U_{B F}<0$ ) or $\mathrm{FB}_{n}$ (for $\left.U_{B F}>0\right)$ with the integer $n \approx\left|U_{B F}\right| / U_{B B}$ can form. In recent experiments [13, 14] complexes of the type $\mathrm{FB}_{n}$ with $n$ as large as $n=3$ may have played an important role.

Depending on the parameters, the effective interaction between composite fermions can be tuned to become attractive or repulsive. In the case of effective attractive interaction, a p-wave superfluid of pairs of composite fermions will form [6, 7]. For effective repulsive interactions and low filling of composite fermions the ground state is a Fermi liquid while near half filling insulating states may appear [10]. For clarity, we will limit ourselves here to the case of composite fermions forming a weakly interacting Fermi liquid with a well defined Fermi surface.

A pure quasi-molecular phase $\mathrm{FB}_{n}$ will only exist when the number of bosons $\mathrm{N}_{B}$ and fermions $\mathrm{N}_{F}$ is commensurate, i.e. $N_{B}=n N_{F}$. Conversely, in the case of a pure phase of composites of the type $\mathrm{FB}_{n}$ the number of bosons must be $N_{B}=N \cdot k-N_{F} \cdot n$, with the integer $k \geq n[23]$ and $N$ standing for the total number of the optical lattice sites. Free fermions or bosons will be present when these conditions are not fulfilled, or when going away from the strongly interacting regime, where the composites can partly dissociate. Then two types of Fermi surfaces can coexist — one for the composites and the other for the original fermions. Moreover, a portion of the B-bosons can form a BEC 24].

Here we will consider the limit of low density of the composites, so that the size of one molecule $a_{m}$ is much less than the average distance $r_{o}$ between them. In this case the description becomes quite straightforward in terms of the centers of mass of the composites only. As $a_{m} / r_{o}$ increases and eventually becomes comparable to 1 , the composite fermions will dissociate according to the scenario [24]. 


\section{B. Detection in weakly interacting Bose-Fermi mixtures}

In the weakly interacting Bose-Fermi mixture without composites present, practically all important information can be deduced by imaging the bosonic $n_{B}(\mathbf{q})$ and fermionic $n_{F}(\mathbf{q})$ quasimomentum distributions. In this method, the quasimomentum distribution is first mapped to the momentum distribution by removing the lattice potential on a time scale which is slow with respect to single particle physics but fast compared to quasimomentum changing collisions. After subsequent free expansion for a time $t$ the quasimomentum distribution is detected by imaging the density [25, 26]. Apart from unimportant multiplicative factors, $n_{B}(\mathbf{q}) \approx \int d \mathbf{x} \exp (-i \mathbf{q x})\left\langle\psi_{B}^{\dagger}(\mathbf{x}) \psi_{B}(0)\right\rangle$ and $n_{F}(\mathbf{q}) \approx$ $\int d \mathbf{x} \exp (-i \mathbf{q x})\left\langle\psi_{F}^{\dagger}(\mathbf{x}) \psi_{F}(0)\right\rangle$, where $\psi_{B}(\mathbf{x})$ and $\psi_{F}(\mathbf{x})$ are the bosonic and fermionic field operators, respectively, just before the lattice was removed and the averaging $\langle\ldots\rangle$ is taken over the corresponding state in the lattice. In the weakly interacting regime, the bosonic quasi-momentum distribution reflects the off-diagonal long range order (ODLRO) inherent in the one-particle density matrix $\rho_{B}\left(\mathbf{x}, \mathbf{x}^{\prime}\right)=\left\langle\psi_{B}^{\dagger}(\mathbf{x}) \psi_{B}\left(\mathbf{x}^{\prime}\right)\right\rangle$, resulting in a very small spread of quasi-momenta. The fermionic oneparticle density matrix $\rho_{F}\left(\mathbf{x}, \mathbf{x}^{\prime}\right)=\left\langle\psi_{F}^{\dagger}(\mathbf{x}) \psi_{F}\left(\mathbf{x}^{\prime}\right)\right\rangle$ features Friedel oscillations which are due to the sharp edge in the Fermi distribution $n_{F}(\mathbf{q})$ (at $T=0$ ): $n_{F}(\mathbf{q}) \approx 1$ for $|\mathbf{q}|<q_{F}$ and $n_{F}(\mathbf{q}) \approx 0$ for $|\mathbf{q}|>q_{F}$, where $q_{F}$ stands for the Fermi wavevector.

\section{Direct detection of composites}

The method described above is not applicable to composite fermions, since they typically dissociate during the expansion. However, the center of mass momentum $\mathbf{Q}$ of a $\mathrm{FB}_{n}$ atomic complex is conserved during time of flight. Therefore the momenta of all its $\mathrm{n}+1$ constituents after expansion are directly correlated and add up to $\mathbf{Q}$. We point out that the observation of such correlations constitutes direct evidence for the existence of the composites. In the following we will describe how these correlations can be used to directly detect the (quasi)momentum distribution of the composites in the lattice.

For an ultracold gas that contains many composites, the fermionic (bosonic) momentum distributions, i.e. the absorption images after time of flight, represent the momenta of many fermions (bosons). Consequently, the correlations between the constituents originating from one composite are not easily visible, since it is not clear which of the many bosons and fermions made up one composite. The correlations can therefore not be determined from the form of the momentum distributions but only from the atomic shot noise visible in them. Since the correlations are between one fermion and $n$ bosons, the corresponding correlator is $\left\langle\delta n_{F}\left(\mathbf{X}_{1}\right) \delta n_{B}\left(\mathbf{X}_{2}\right) \ldots \delta n_{B}\left(\mathbf{X}_{n+1}\right)\right\rangle$.
To determine the amount of correlation, this correlator must be integrated over $n+1$ coordinates under the constraint that the center of mass momentum $\mathbf{Q}$ of the constituents is fixed:

$$
\begin{gathered}
I_{n+1}(\mathbf{Q})=\int d \mathbf{q}_{2} \ldots d \mathbf{q}_{n+1} \\
\left\langle: \delta n_{F}\left(\mathbf{q}_{1}\right) \delta n_{B}\left(\mathbf{q}_{2}\right) \ldots \delta n_{B}\left(\mathbf{q}_{n+1}\right):\right\rangle,
\end{gathered}
$$

with $\mathbf{Q}=\mathbf{q}_{1}+\mathbf{q}_{2}+\ldots+\mathbf{q}_{n+1}$ and $\mathbf{q}_{1}=m_{F} \mathbf{X}_{1} / t, \mathbf{q}_{2}=$ $m_{B} \mathbf{X}_{2} / t, \ldots, \mathbf{q}_{n+1}=m_{B} \mathbf{X}_{n+1} / t$. Here : ... : implies the normal order of the operators - when all the creation operators stay to the left of the annihilation ones. The averaging $\langle\ldots\rangle$ is performed over a particular realization of a many body eigenstate $|\Psi\rangle$ and, then, over many realizations. In appendices $\mathrm{A}$ and $\mathrm{B}$ we show that the amount of correlation $I_{n+1}(\mathbf{Q})$ is proportional to the number of composites with given quasi-momentum $\mathbf{Q}$, that is

$$
I_{n+1}(\mathbf{Q}) \sim \tilde{\rho}_{C F}(\mathbf{Q})-C,
$$

where $\tilde{\rho}_{C F}(\mathbf{Q})=\left\langle c^{\dagger}(\mathbf{Q}) c(\mathbf{Q})\right\rangle$ and $c^{\dagger}(\mathbf{Q}), c(\mathbf{Q})$ are the composite fermion creation and annihilation operators in the momentum space and $C$ is a constant term. To summarize, the quasimomentum distribution $\tilde{\rho}_{C F}(\mathbf{Q})$ of the composites can be found by measuring the correlations between experimental absorption images according to the equation (2).

The relation (3) is proved rigorously in appendices A and B. It is instructive to follow the main steps of the proof: The operators

$$
\begin{aligned}
& \delta n_{F}(\mathbf{X})=\psi_{F}^{\dagger}(\mathbf{X}) \psi_{F}(\mathbf{X})-\left\langle\Psi\left|\psi_{F}^{\dagger}(\mathbf{X}) \psi_{F}(\mathbf{X})\right| \Psi\right\rangle, \\
& \delta n_{B}(\mathbf{X})=\psi_{B}^{\dagger}(\mathbf{X}) \psi_{B}(\mathbf{X})-\left\langle\Psi\left|\psi_{B}^{\dagger}(\mathbf{X}) \psi_{B}(\mathbf{X})\right| \Psi\right\rangle,
\end{aligned}
$$

appearing in equation (2) are expressed as functions of the onsite fermionic and bosonic creation and annihilation operators $f_{i}^{\dagger}, f_{i}, b_{i}^{\dagger}, b_{i}$, with $i$ being the site index, in the lattice before expansion with the help of eqs. A4 A5 A33). Inserting these expressions into eq.(2) yields sums over terms with $2(n+1)$ creation and annihilation operators on different sites. Many of these can be set to zero, resulting in

$$
I_{n+1}(\mathbf{Q}) \sim \sum_{i j} \mathrm{e}^{i \mathbf{Q}\left(\mathbf{x}_{i}-\mathbf{x}_{j}\right)}\left\langle\left[c_{i}^{\dagger} c_{j}-\delta_{i j} c_{i}^{\dagger} c_{i}\right]\right\rangle
$$

where $c_{i}^{\dagger}, c_{i}$ are the onsite composite fermion creationannihilation operators as defined in eq. (B1) of the Appendix $\mathbb{B}$ and $\mathbf{x}_{i}$ denotes the site $i$ coordinates. The first term in brackets in eq.(6) is $\tilde{\rho}_{C F}(\mathbf{Q})$ : as can be noticed, after introducing the composite Fermi operators in the Fourier space $c(\mathbf{Q}) \sim \sum_{i} \exp \left(-i \mathbf{Q} \mathbf{x}_{i}\right) c_{i}$, one finds equation (3). While $\tilde{\rho}_{C F}(\mathbf{Q})$ gives the occupation number of composites with quasimomentum $\mathbf{Q}$, the second term is the constant $C$ which takes care of the normalization $\int d \mathbf{Q} I_{n+1}(\mathbf{Q})=0$. The last relation follows from the observation that the integral $\int d \mathbf{Q} I_{n+1}(\mathbf{Q})$ is exactly $\left\langle\delta N_{F}:\left(\delta N_{B}\right)^{n}:\right\rangle$ where $\delta N_{B, F}$ denote fluctuations of the 
total numbers of bosons and fermions $N_{B, F}$, respectively. Since $N_{F}$ is diagonal on any many-body eigenstate $|\Psi\rangle$, such a mean is exactly zero for any particular realization of $|\Psi\rangle$, that is $\delta N_{F}|\Psi\rangle=0$. Both contributions in eqs. 36 can easily be distinguished because they have very different structures: the first one is concentrated within the Fermi surface of the composite fermions and the second is spread uniformly over all momenta in the first Brillouin zone.

In the case of the complexes $\mathrm{FB}_{n}$ ( see the discussion in Appendix Abelow eqs. A9 A10) the only change is in the definition of the momentum $\mathbf{Q}$ in eqs.(2). It becomes $\mathbf{Q}=\mathbf{q}_{1}-\mathbf{q}_{2}-\ldots-\mathbf{q}_{n+1}$.

As discussed in ref. 24], decreasing the F-B interaction $U_{B F}$ will eventually result in coexistence of three types of quasi-particles: some portion of free B-bosons and F-fermions and the composites. In order to detect the composites in this case, the noise must be measured on top of signals from the structured backgrounds $\left\langle n_{F}(\mathbf{q})\right\rangle,\left\langle n_{B}(\mathbf{q})\right\rangle$ in the fermionic and bosonic channels.

\section{Correlated versus uncorrelated noise}

Here we discuss the detection requirements under the assumption that the atomic quantum and shot-to-shot noise is dominant. The above results indicate that measuring the noise in the density correlator of the $n+1$ order can provide a one-particle density matrix for the centers of mass of the composites. It is important, however, that the uncorrelated noise due to photon shot noise, inaccuracy $\Delta$ in determination of the number of particles in each detection bin, etc., is much less than the correlated noise in one shot. Otherwise, many shots $S$ are required in order to reduce the effect of the uncorrelated noise (by the factor $\sim 1 / \sqrt{S}$ ). Here we will show that, while for diatomic composites the uncorrelated noise (with respect to the correlated one) is insignificant for large enough numbers $N_{F} \sim N_{B}$ (decays as $1 / \sqrt{N_{F}}$ ), for multi-atomic composites $\mathrm{FB}_{n}$ with $n=3, .$. it grows as $\sim N_{F}^{n / 2-1}$, with the case $n=2$ being marginal. This makes the direct scheme of measuring the density matrix of the $n>2$ composites impractical because the required number of shots grows as $S \sim N_{F}^{n-2}$. This effect is caused by a strong increase of the phase space with $n$ when the composites are dissociated as a result of the lattice release.

In what follows we will normalize the correlated and uncorrelated noises by the corresponding measured signal

$$
\begin{aligned}
I_{s i g}=\int & d \mathbf{X}_{2} \ldots d \mathbf{X}_{n+1}\left\langle n_{F}\left(\mathbf{Q}-\mathbf{q}_{\mathbf{2}}-\ldots-\mathbf{q} \mathbf{n}+\mathbf{1}\right)\right\rangle \\
& \left\langle n_{B}\left(\mathbf{q}_{2}\right)\right\rangle \ldots\left\langle n_{B}\left(\mathbf{q}_{n+1}\right)\right\rangle
\end{aligned}
$$

where $\mathbf{q}_{k}=m_{B} \mathbf{X}_{k} / t$. This integral can be estimated as $I_{\text {sig }} \approx N_{B}^{n} N_{F} / N_{\text {bin }}=n^{n} N_{F}^{n+1} / N_{\text {bin }}$, where we consider exact matching $N_{B}=n N_{F}$ and $N_{\text {bin }}$ stands for the total number of the detection bins. In the following estimates we will be using the relations $N_{B} \sim N_{F} \sim N$, so that the actual numbers of particles can be replaced by the total number of sites (provided the densities are finite).
The uncorrelated noise is caused by technical inaccuracies $\Delta\left(\mathbf{X}_{i}\right)$ in the detection of the number of, e.g., fermions in each detection bin. The effect of such noise becomes critically magnified for large $n$ even under the condition that $\left|\Delta\left(\mathbf{X}_{i}\right)\right|$ is much smaller than a typical quantum fluctuation $\sim \sqrt{N_{F} / N_{\text {bin }}} \sim \sqrt{N / N_{\text {bin }}} \gg 1$ of the number of detected particles per each bin. We note that, under the ideal condition $\Delta\left(\mathbf{X}_{i}\right)=0$, quantum fluctuations of the particles number in each bin do not give rise to any uncorrelated noise in the correlator (2). This is due to the fact that the center of mass momenta of the composites are good quantum numbers - in contrast to the momenta of the constituents - and therefore the corresponding quantity, the density of the composites represented by the operator in eq.(2) does not fluctuate in any particular many-body eigenstate $|\Psi\rangle$. If, however, the measurement is done with an error $\Delta\left(\mathbf{X}_{i}\right)$, the operator determining the correlator (2) acquires the contribution $\Delta \hat{I}=\int d \mathbf{X}_{2} \ldots d \mathbf{X}_{n+1} \Delta\left(\mathbf{X}_{1}\right) \delta n_{B}\left(\mathbf{X}_{2}\right) \ldots \delta n_{B}\left(\mathbf{X}_{n+1}\right)$, which can be considered as a fair estimate of the total error under the condition $|\Delta| \ll \sqrt{N_{F} / N_{\text {bin }}}$. Clearly the mean of it is zero, $\langle\Delta \hat{I}\rangle=0$. However, its rms fluctuation $E=\sqrt{\langle\Delta \hat{I} \Delta \hat{I}\rangle} / I_{\text {sig }}$ (normalized by $\left.I_{\text {sig }}\right)$ is not zero. We estimate: $\langle\Delta \hat{I} \Delta \hat{I}\rangle \sim$ $\left\langle\Delta^{2}\right\rangle\left(\int d \mathbf{X}_{1} \int d \mathbf{X}_{2}\left\langle\delta n_{B}\left(\mathbf{X}_{1}\right) n_{B}\left(\mathbf{X}_{2}\right)\right\rangle\right)^{n} \sim\left\langle\Delta^{2}\right\rangle N^{n}$, where we have taken into account that the quantum fluctuation of the number of bosons in each bin is $\sim$ $\sqrt{N / N_{b i n}}$ and we have considered no correlations between the bins (and omitted factors dependent on $n$ like $n !, n^{n}$, etc.) . Given this estimate and the one for $I_{\text {sig }}$ in eq.(17), we find

$$
E=\frac{\langle|\Delta|\rangle N_{\mathrm{bin}}}{N^{1+n / 2}},
$$

where $\langle|\Delta|\rangle$ stands for the rms-fluctuations of $\Delta\left(\mathbf{X}_{i}\right)$.

The quantity $E$ charaterizes the uncorrelated noise contribution in one shot. If $S$ shots are performed, it is reduced as $E \rightarrow E / \sqrt{S}$ so that, in principle, the effect of the uncorrelated noise can be reduced below the correlated contribution $I_{n+1} / I_{\text {sig }}$ after taking a sufficient amount of the shots. In reality, the required $S$ must scale not faster than $S \sim \mathcal{O}(1)$ with $N_{F}$. The correlated contribution (6) is given just by the total number of the composites $\sim N_{F}$ in each bin: $I_{n+1} \sim$ $N_{F} / N_{b i n} \sim N / N_{b i n}$. Hence, $I_{n+1} / I_{\text {sig }} \approx N^{-n}$ and $\Delta I / I_{n+1} \sim\langle|\Delta|\rangle N_{b i n} N^{n / 2-1} / \sqrt{S}$. Thus, the required number of shots is

$$
S \gg|\Delta|^{2} N_{\text {bin }}^{2} N^{n-2} .
$$

For $n=1$, this condition can be achieved for large enough $N$ and not very large number of bins $N_{\text {bin }}$. For $n=2$, the condition is marginally reachable, and, for $n>2$, it becomes impossible to fulfill.

It is worth mentioning that, in the case of composite bosons, say, $\mathrm{AB}_{n}$, where A labels some boson of a kind different from B, the condition (9) becomes less strict. Indeed, $I_{n+1}$ (2) in this case acquires an additional factor 
$\sim N$ due to the ratio of the correlation volumes of a boson and a fermion. Indeed, the sum $\sum_{i j} \mathrm{e}^{i \mathbf{Q}\left(\mathbf{x}_{i}-\mathbf{x}_{j}\right)}\left\langle c_{i}^{\dagger} c_{j}\right\rangle$ can be estimated as $\sim N \xi^{3}$, where $\xi$ is a typical distance on which the correlator $\left\langle c_{i}^{\dagger} c_{j}\right\rangle$ becomes zero. For fermions it is given by the interparticle separation $r_{o}$. For bosons in BEC, it is the size of the lattice and one finds $I_{n+1} \sim N^{2} / N_{b i n}$. Accordingly, keeping the signal and the uncorrelated noise estimates the same, we find the number of the required shots $S \gg|\Delta|^{2} N_{\text {bin }}^{2} N^{n-4}$. This implies that for composite bosons the method described above can be used if $n \leq 4$, that is the total number $n+1$ of the constituents should not exceed five.

Concluding this section, we mention that performing shot-to-shot averaging should be done with care, because, otherwise, the noise scaling with $N$ can become even stronger. The operators of fluctuations $\delta n_{F}=$ $n_{F}-\left\langle n_{F}\right\rangle, \delta n_{B}=n_{B}-\left\langle n_{B}\right\rangle, \delta n_{A}=n_{A}-\left\langle n_{A}\right\rangle$ are defined with respect to averaging over a particular ground state characterized by given total numbers of atoms $N_{F}, N_{B}, N_{A}$. In other words, the average number of atoms $\left\langle n_{F}\right\rangle,\left\langle n_{B}\right\rangle,\left\langle n_{A}\right\rangle$ per each bin must be determined in each shot. Then, the final value $I_{n+1}$ can be averaged over the shots. Otherwise, shot-to-shot fluctuations of $N_{F, B, A}$ which typically scale at least as $\sqrt{N_{F, B, A}}$, respectively, will introduce stronger noise if the means $\left\langle n_{F}\right\rangle,\left\langle n_{B}\right\rangle,\left\langle n_{A}\right\rangle$ are understood as the total ensemble means. In the latter case the difference between the mean in one particular realization and the total one serves as an additional uncorrelated noise. Then, one can estimate $\Delta \sim \sqrt{N} / N_{\text {bin }}$ and, accordingly, find $\Delta I / I_{n+1} \sim$ $N^{(n-1) / 2} / \sqrt{S}$, so that the required number of shots scales as $S \sim N^{n-1}$, implying that the detection of the composite fermions with $n>1$ is not possible. Similarly, for the composite bosons: $\Delta I / I_{n+1} \sim N^{(n-3) / 2} / \sqrt{S}$, and the detection for $n>3$ becomes impossible.

\section{INDIRECT INDICATION OF THE COMPOSITE FERMIONS IN THE BOSONIC DENSITY-DENSITY NOISE CORRELATOR}

Here we discuss the situation when all bosons are bound to be the constituents of the composite fermions so that there is no ODLRO in the lattice. The question is how the bosonic density-density correlator

$$
I_{b b}(\mathbf{Y})=\int d \mathbf{X}\left\langle\Psi\left|: n_{B}(\mathbf{X}+\mathbf{Y} / 2) n_{B}(\mathbf{X}-\mathbf{Y} / 2):\right| \Psi\right\rangle
$$

(taken in the normal order) is affected by such state. Below we show that (10) exhibits the Bragg structure similar to that observed in the Bose Mott insulator (MI) [18, 19]. The relations (B6 B7) in the physical space of the composites (B2) allow expressing the correlator (10) as the mean over the operator

$$
\begin{aligned}
\hat{I}_{b b}(\mathbf{Y}) & =n^{2} \sum_{i j}\left(1+\mathrm{e}^{i\left(m_{B} \mathbf{Y} / t\right)\left(\mathbf{x}_{i}-\mathbf{x}_{j}\right)}\right) c_{i}^{\dagger} c_{j}^{\dagger} c_{j} c_{i} \\
& +n(n-1) \sum_{i} c_{i}^{\dagger} c_{i}
\end{aligned}
$$

where the last term, which is $\sim o(1 / N)$ with respect to the first one, can be ignored. Taking the means and replacing $\left\langle c_{i}^{\dagger} c_{j}^{\dagger} c_{j} c_{i}\right\rangle=\nu^{2}\left(1-\delta_{i j}\right)$, with $\nu$ being the average onsite population of fermions, we find that eq. (11) has exactly the same structure observed for the bosonic Mott insulator [18, 19]: the term $\sim \exp \left[i\left(m_{F, B} \mathbf{Y} / t\right)\left(\mathbf{x}_{i}-\mathbf{x}_{j}\right)\right]$ is the correlated contribution [15] which peaks as long as $\mathbf{Y}$ matches the positions of the Bragg peaks (in other words, $\mathbf{Y}=0$ modulo the primitive vectors of the reciprocal lattice). It is important that this structure exists for any filling factor of the composites $\nu \leq 1$, that is, it is not a sole property of the Mott insulating ground states.

Regarding the fermionic correlator

$$
I_{f f}(\mathbf{Y})=\int d \mathbf{X}\left\langle\Psi\left|: n_{F}(\mathbf{X}+\mathbf{Y} / 2) n_{F}(\mathbf{X}-\mathbf{Y} / 2):\right| \Psi\right\rangle
$$

we note that it must exhibit the antibunching peaks, that is, $I_{f f}(\mathbf{Y})=0$ for $\mathbf{Y}=0$ (modulo the primitive vectors of the reciprocal lattice) regardless of the nature of the ground state because the normal product $: n_{F}(\mathbf{X}) n_{F}(\mathbf{X})$ : is zero (due to the Pauli principle and the periodicity of the lattice). This can be verified directly with the help of eqs. (A4A3). Then, Eq. (12) becomes a periodic function $I_{f f}(\mathbf{Y})$, with the periods determined by the primitive vectors of the reciprocal lattice, and one finds $I_{f f}(0) \sim \int d \mathbf{q} \sum_{i j k l} \exp \left(i \mathbf{q}\left(\mathbf{x}_{i}+\mathbf{x}_{k}-\right.\right.$ $\left.\left.\mathbf{x}_{j}-\mathbf{x}_{l}\right)\right)\left\langle f_{i}^{\dagger} f_{k}^{\dagger} f_{l} f_{j}\right\rangle=0$ due to the Fermi statistics: $f_{l} f_{j}+f_{j} f_{l}=0$.

The physical reason why the bosonic correlator (10) shows bunching peaks (11) in a strongly interacting mixture stems from a very general principle, which is best explained by starting from the opposite case. In any non-interacting pure quantum mechanical state the correlator $\left\langle\Psi_{0}\left|\delta n_{B}(\mathbf{X}) \delta n_{B}(\mathbf{Y})\right| \Psi_{0}\right\rangle$ (not taken in the normal order!) must be exactly zero. To understand this one must remember that the density operators in the far zone are equivalent to the (quasi-)momentum operators in the lattice and that non-interacting systems are characterized by well defined quasi-momenta. Thus, there are no fluctuations in each particular manybody (non-interacting) eigenstate $\left|\Psi_{0}\right\rangle$ and the correlator $\left\langle\Psi_{0}\left|\delta n_{B}(\mathbf{X}) \delta n_{B}(\mathbf{Y})\right| \Psi_{0}\right\rangle$ is zero. In other words the operator (5) is zero on space of states $\left|\Psi_{0}\right\rangle$ of the ideal systems: that is, $\delta n_{B}(\mathbf{X})\left|\Psi_{0}\right\rangle=0$. In the thermodynamical limit at low $T$ the normal ordering in eq. (10) can be ignored for bosons (as opposed to fermions!). Consequently, a weakly interacting BEC of B-atoms will lead to essentially uniform distributions in eq.(10) 27. 
Meanwhile in a quasi-molecular phase of strongly interacting Bose-Fermi mixtures the momenta of the original bosons are undefined in each pure many-body state $|\Psi\rangle$ in complete analogy with the MI. Accordingly, the densities in the far zone fluctuate strongly and produce the bunching behaviour in (11). In this case quantum fluctuations are responsible for the Hanbury Brown and Twiss effect. Setting it more technically, in each manybody eigenstate the correlator $\left\langle\Psi\left|\delta n_{B}(\mathbf{X}) \delta n_{B}(\mathbf{Y})\right| \Psi\right\rangle$ is proportional to the interaction vertex [28] and, if the interaction is small, the correlator is, practically, zero. In general, having a strong interaction regime is the requirement for observing the distinct patterns (11) in pure states. This condition is more general than the one for having energy gap with respect to single-particle excitations 18. In the state mentioned in Sec IC where all three types of quasi-particles are present, such a gap is zero and the composites can still be well defined. Accordingly, the Hanbury Brown and Twiss pattern (11) should be observable. Obviously, some care should be taken in order not to confuse the pattern due to the composites with that caused by thermal fluctuations 27].

\section{CONCLUSION AND ACKNOWLEDGEMENT}

We have suggested the extended density-density correlator method for the direct imaging of the composites in optical lattice and have evaluated its feasibility for multiatomic fermionic and bosonic complexes. The method is expected to work well for fermionic composites consisting of less than four atoms and for bosonic ones consisting of less than six atoms. Above these numbers, the uncorrelated noise becomes too large.

As an indirect method for detecting the composites, measuring the bosonic density-density correlators must reveal the typical Hanbury Brown and Twiss structure first observed for the bosonic Mott insulator. We point out that this observation is a generic consequence of strong interactions in a two-component mixture in optical lattices.

We thank M. Köhl for a critical reading of the manuscript. This work was supported by NSF grant PHY-0426814, PSC-CUNY grant PSC-66556-0037.

\section{APPENDIX A: STRONGLY BOUND FB AND F $\bar{B}$ PAIRS}

Here we will give a more extended treatment of the FB case discussed in the introduction. It is important that the correlator (11) turns out to be proportional to the Fourier representation of the in-situ two-body density matrix $\left\langle\psi_{F}^{\dagger}\left(\mathbf{x}_{1}\right) \psi_{B}^{\dagger}\left(\mathbf{x}_{2}\right) \psi_{F}\left(\mathbf{y}_{1}\right) \psi_{B}\left(\mathbf{y}_{2}\right)\right\rangle$ with respect to the coordinate $\mathbf{x}_{1}-\mathbf{y}_{1}$. As a matter of fact, since a boson can only be found close to a fermion, one can set $\mathbf{x}_{1}=\mathbf{x}_{2}, \mathbf{y}_{1}=\mathbf{y}_{2}$ in this correlator without affecting its low energy properties (as long as $a_{m} / r_{o} \ll 1$ ).

In the following we will justify the statement $I_{C F}(\mathbf{Q}) \sim$ $\tilde{\rho}_{C F}(\mathbf{Q})$. We assume that the wavefunction of relative motion $\Phi(\mathbf{r})$ of a boson and a fermion in a pair is of the s-wave type $\Phi(\mathbf{r}) \approx \exp \left(-|\mathbf{r}| / a_{m}\right) / a_{m}^{3 / 2}$ with $a_{m}$ being of the order of the lattice constant. [In the case of the non-s-wave BF pairing, the correlator (11) vanishes after integration over, say, $\mathbf{q}_{2}$ with $\mathbf{Q}$ kept constant. We do not consider such an exotic possibility]. We will see in the following that once $\left|\mathbf{Q} a_{m}\right| \ll 1$, the extent of the wavefunction $a_{m}$ has no effect on $I_{C F}(\mathbf{Q})$ or $\tilde{\rho}_{C F}(\mathbf{Q})$. In order to calculate $I_{C F}(\mathbf{Q})$, we expand the fermionic and bosonic operators in the optical lattice as

$$
\begin{aligned}
& \psi_{F}(\mathbf{x})=\sum_{i} W_{0}\left(\mathbf{x}-\mathbf{x}_{i}\right) f_{i}, \\
& \psi_{B}(\mathbf{x})=\sum_{i} W_{0}\left(\mathbf{x}-\mathbf{x}_{i}\right) b_{i},
\end{aligned}
$$

where $W_{0}\left(\mathbf{x}-\mathbf{x}_{i}\right)$ stands for the Wannier function located at $i$ th site. For simplicity, we consider $W_{0}(\mathbf{x})$ to be the same for fermions and bosons. Upon free expansion of a particle of mass $m$, the Wannier function in the far zone becomes (apart from a numerical coefficient)

$$
W_{t}\left(\mathbf{X}-\mathbf{x}_{i}\right)=\left(\frac{m}{t}\right)^{3 / 2} \mathrm{e}^{-i \mathbf{q} \mathbf{x}_{i}+i m \mathbf{X}^{2} / 2 t} \tilde{W}(\mathbf{q})
$$

where $\mathbf{q}=m \mathbf{X} / t$ (we employ units in which $\hbar=1$ ) and $\tilde{W}(\mathbf{q})=\int d \mathbf{x}^{\prime} \exp \left(-i \mathbf{q} \mathbf{x}^{\prime}\right) W_{0}\left(\mathbf{x}^{\prime}\right)$ stands for the Fourier transform of the Wannier function. In the far zone the operators A1A2 become

$$
\begin{aligned}
& \psi_{F}(\mathbf{X})=\sum_{i} W_{t}\left(\mathbf{X}-\mathbf{x}_{i}\right) f_{i} \\
& \psi_{B}(\mathbf{X})=\sum_{i} W_{t}\left(\mathbf{X}-\mathbf{x}_{i}\right) b_{i}
\end{aligned}
$$

where the corresponding mass $m_{F}, m_{B}$ is replacing $m$.

Then, substitution of eqs. A4A5 into the definition of the densities in the far zone (45) and employing them in eq. (11) give 


$$
\begin{aligned}
I_{C F}(\mathbf{Q})= & \int d \mathbf{q}_{2} \frac{m_{F}^{3}\left|\tilde{W}\left(\mathbf{Q}-\mathbf{q}_{2}\right) \tilde{W}\left(\mathbf{q}_{2}\right)\right|^{2}}{t^{3}}\left[\left\langle f^{\dagger}\left(\mathbf{Q}-\mathbf{q}_{2}\right) b^{\dagger}\left(\mathbf{q}_{2}\right) f\left(\mathbf{Q}-\mathbf{q}_{2}\right) b\left(\mathbf{q}_{2}\right)\right\rangle-\right. \\
& \left.\left\langle f^{\dagger}\left(\mathbf{Q}-\mathbf{q}_{2}\right) f\left(\mathbf{Q}-\mathbf{q}_{2}\right)\right\rangle\left\langle b^{\dagger}\left(\mathbf{q}_{2}\right) b\left(\mathbf{q}_{2}\right)\right\rangle\right]
\end{aligned}
$$

where $f(\mathbf{q})=\frac{1}{\sqrt{N}} \sum_{i} \exp \left(-i \mathbf{q} \mathbf{x}_{i}\right) f_{i}$ and $b(\mathbf{q})=$ $\frac{1}{\sqrt{N}} \sum_{i} \exp \left(-i \mathbf{q} \mathbf{x}_{i}\right) b_{i}$ are the Fourier representations of the onsite operators. We note that $I_{C F}(\mathbf{Q})$ scales with the expansion radius $R \sim t$ as $\sim 1 / R^{3}$, that is, as though it is just a density of some particle undergoing free expansion.

The momenta involved in $\tilde{W}(\mathbf{q})$ are of the order of the inverse lattice constant $a_{l}$. In fact, as the lattice is being ramped down adiabatically with respect to the single particle states, the Wannier function undergoes a transformation from that corresponding to the deep lattice to the one in the very shallow lattice. In the latter case, $W(\mathbf{x})$ can be found explicitly from the definition (1) of ref. [29], where for the Bloch function one can use just the exponential $\exp (i \mathbf{k x})$. Then, the square of the Fourier transform $|\tilde{W}(\mathbf{q})|^{2}$ trivially becomes the volume of the elementary cell $\Omega_{B Z} \approx a_{l}^{3}$ when $\mathbf{q}$ belongs to the first Brillouin zone and zero otherwise. Accordingly, the integration $\int d \mathbf{q}_{2} \ldots$ in eq. A6 proceeds over the first Brillouin zone $\int_{B Z} d \mathbf{q}_{2} \ldots$ with the factor $\left|\tilde{W}\left(\mathbf{Q}-\mathbf{q}_{2}\right) \tilde{W}\left(\mathbf{q}_{2}\right)\right|^{2}=1$ as long as the typical values of $\mathbf{Q}$ are much smaller than the inverse lattice constant $a_{l}$ (for $\mathbf{Q}$ being outside the first zone, this factor is essentially zero, if the Fermi "sphere" has a volume $\approx Q_{C F}^{3}$ much less than $1 / \Omega_{B Z}$ ). In particular, this implies that the "imaged" composites are localized mostly within the first Brillouin zone similarly to the rampdown procedures [25, 26] used for the atomic imaging. As can be seen, the spreading over the next zone is suppressed by the factor $Q_{C F}^{3} \Omega_{B Z} \ll 1$ due to the small fraction of the phase volume in the integral (A6) which allows access to the center of the next zone.

If bosons and fermions do not bind together, the correlator in eq. tightly bound composites FB in the lattice, the correlator become finite whenever a boson is found within the proximity $a_{m}$ of a fermion. This is indicated in a choice of the physical space

$$
\begin{gathered}
\left|1,\left\{n_{\mathbf{Q}}\right\}\right\rangle=\prod_{\mathbf{Q}}\left(c_{\mathbf{Q}}^{\dagger}\right)^{n_{\mathbf{Q}}}|0\rangle, \\
c_{\mathbf{Q}}^{\dagger}=\int d \mathbf{q} \tilde{\Phi}(\mathbf{q}) f^{\dagger}(\mathbf{Q} / 2+\mathbf{q}) b^{\dagger}(\mathbf{Q} / 2-\mathbf{q}),
\end{gathered}
$$

with $n_{\mathbf{Q}}=0,1$, of weakly interacting composite fermions (in the considered limit $r_{o} \ll a_{m}$ ). It is represented by the composite creation-annihilation operators $c_{\mathbf{Q}}^{\dagger}, c_{\mathbf{Q}}$ of a $\mathrm{BF}$ pair with total momentum $\mathbf{Q}$ in terms of the Fourier components of the fermionic and bosonic operators and of the relative wavefunction $\tilde{\Phi}(\mathbf{q})$. It is important that the low energy properties do not depend on details of
$\tilde{\Phi}(\mathbf{q})$ in the limit of low density of the composites. This can be seen by direct calculation of eq. A6 over the state (A7). Specifically, the state $f\left(\mathbf{Q}-\mathbf{q}_{2}\right) b\left(\mathbf{q}_{2}\right)\left|1,\left\{n_{\mathbf{Q}}\right\}\right\rangle$ contains contribution $\tilde{\Phi}\left(\mathbf{Q} / 2-\mathbf{q}_{2}\right)|0\rangle$ coming from the action of $f\left(\mathbf{Q}-\mathbf{q}_{2}\right) b\left(\mathbf{q}_{2}\right)$ on the term describing just single composite $c_{\mathbf{Q}}^{\dagger}|0\rangle$ without affecting the others. It also contains the exchange terms involving pairs of the composites with different momenta. These exchange terms, however, can clearly be neglected because they contribute factors $\left(a_{m} / r_{o}\right)^{3} \ll 1$. Thus, neglecting these terms, we find $\int_{B Z} d \mathbf{q}_{2}\left\langle\left\{n_{\mathbf{Q}}\right\}, 1\right| f^{\dagger}\left(\mathbf{Q}-\mathbf{q}_{2}\right) b^{\dagger}\left(\mathbf{q}_{2}\right) f(\mathbf{Q}-$ $\left.\mathbf{q}_{2}\right) b\left(\mathbf{q}_{2}\right)\left|1,\left\{n_{\mathbf{Q}}\right\}\right\rangle=\int_{B Z} d \mathbf{q}_{2}\left|\tilde{\Phi}\left(\mathbf{q}_{2}\right)\right|^{2} n_{\mathbf{Q}}=$ $\left\langle\left\{n_{\mathbf{Q}}\right\}, 1\left|c^{\dagger}(\mathbf{Q}) c(\mathbf{Q})\right| 1,\left\{n_{\mathbf{Q}}\right\}\right\rangle$, since $\int_{B Z} d \mathbf{q}_{2}\left|\tilde{\Phi}\left(\mathbf{q}_{2}\right)\right|^{2}=1$ as the normalization condition. Thus, in the physical space A7/A8) the operator $\int_{B Z} d \mathbf{q}_{2} f^{\dagger}(\mathbf{Q}-$ $\left.\mathbf{q}_{2}\right) b^{\dagger}\left(\mathbf{q}_{2}\right) f\left(\mathbf{Q}-\mathbf{q}_{2}\right) b\left(\mathbf{q}_{2}\right)$ is equal to $c^{\dagger}(\mathbf{Q}) c(\mathbf{Q})$ and its averaging produces the momentum distribution $\tilde{\rho}_{C F}(\mathbf{Q})=\left\langle\left\{n_{\mathbf{Q}}\right\}, 1\left|c^{\dagger}(\mathbf{Q}) c(\mathbf{Q})\right| 1,\left\{n_{\mathbf{Q}}\right\}\right\rangle$ of the composite fermions as discussed in the Introduction below eq.(11).

As can be directly checked, the uncorrelated contribution $\int_{B Z} d \mathbf{q}_{2}\left\langle f^{\dagger}\left(\mathbf{Q}-\mathbf{q}_{2}\right) f\left(\mathbf{Q}-\mathbf{q}_{2}\right)\right\rangle\left\langle b^{\dagger}\left(\mathbf{q}_{2}\right) b\left(\mathbf{q}_{2}\right)\right\rangle$ in eq. (A6) is independent of $\mathbf{Q}$ as long as $|\mathbf{Q}| \ll a_{m}^{-1}$ and, thus, can be replaced by a constant $C$. This constant can be restored from the normalization on the density of fermions (and bosons). Finally, the expression (10) becomes $I_{C F}(\mathbf{Q}) \sim \tilde{\rho}_{C F}(\mathbf{Q})-C$, which is a particular case of a more general eq. (3) discussed in the section IIC

We also comment on the case of purely repulsive interactions when the pairing occurs between fermions and bosonic holes [7]. The corresponding physical space, then, becomes

$$
\begin{gathered}
\left|1,\left\{n_{\mathbf{Q}}\right\}\right\rangle=\prod_{\mathbf{Q}}\left(c_{\mathbf{Q}}^{\dagger}\right)^{n_{\mathbf{Q}}} \prod_{i}\left(b_{i}^{\dagger}\right)^{k}|0\rangle \\
c_{\mathbf{Q}}^{\dagger}=\int d \mathbf{q} \tilde{\Phi}(\mathbf{q}) f^{\dagger}(\mathbf{Q} / 2+\mathbf{q}) b(\mathbf{q}-\mathbf{Q} / 2),
\end{gathered}
$$

where $k=1,2, .$. and $\tilde{\Phi}(\mathbf{q})$ is now a wavefunction of relative motion of a fermion and bosonic hole. Accordingly, the total momentum $\mathbf{Q}$ is now the difference of the momenta carried by the fermion $\mathbf{Q} / 2+\mathbf{q}$ and by the bosonic hole $\mathbf{q}-\mathbf{Q} / 2$. Then, as discussed in the Introduction, the momentum $\mathbf{Q}$ in eq. (11) is to be replaced by the difference of the momenta: $\mathbf{Q}_{-}=\mathbf{q}_{1}-\mathbf{q}_{2}$. 


\section{APPENDIX B: THE PHYSICAL HILBERT SPACE FOR STRONGLY BOUND COMPLEXES FB ${ }_{n}$}

Insensitivity of the low energy physics to the internal structure of the composites discussed in the Appendix A for the case FB (as an example) allows a simplification of the analysis by projecting the Hamiltonian as well as any measurable quantity into states having either no particles on a site or $1+n$ particles - one fermion and $n$ bosons. In other words, the constituents of a composite are located at the same site and the probability to find an unbound boson or fermion on another site is vanishingly small. This means that the physical operators are

$$
c_{i}=\frac{f_{i} b_{i}^{n}}{\sqrt{n !}}, \quad c_{i}^{\dagger}=\frac{f_{i}^{\dagger} b_{i}^{\dagger n}}{\sqrt{n !}}
$$

Then, the physical Hilbert space is represented by the basis

$$
\left|n,\left\{n_{i}\right\}\right\rangle=\prod_{i}\left(c_{i}^{\dagger}\right)^{n_{i}}|0\rangle,
$$

where the product is taken over all lattice sites and $n_{i}$ can take only two values $n_{i}=0,1$. As can be easily checked, the annihilation and creation operators of the composite fermions obey the standard anti-commutation relation $\left[c_{i}, c_{j}^{\dagger}\right]_{+}=\delta_{i j}$ in the space (B2).

The projection of the measured quantities into the space (B2) can be done by considering processes leading to jumps of the composite fermions only. If the composites are strongly bound and there are no free bosons or fermions, this is a very accurate approximation. Then, we ignore $b_{i}^{\dagger} b_{j}$ and $f_{i}^{\dagger} f_{j}$ acting on $\left|n,\left\{n_{i}\right\}\right\rangle$ unless $i=j$ which gives onsite local populations determined by presence or absence of a composite on the site $i$. So, in general

$$
\begin{aligned}
b_{i}^{\dagger} b_{j} & =n c_{i}^{\dagger} c_{i} \delta_{i j}, \\
f_{i}^{\dagger} f_{j} & =c_{i}^{\dagger} c_{i} \delta_{i j} .
\end{aligned}
$$

Concerning the quartic operators $f_{i}^{\dagger} f_{j} b_{k}^{\dagger} b_{l}$ in eq.(1) which are important for the detection of the FB quasimolecules, they must be set to zero (if acting on $\left|1,\left\{n_{i}\right\}\right\rangle$ ) unless $i=j, k=l$ or $i=k, j=l$. For $n=1$, the following relation holds

$$
f_{i}^{\dagger} f_{j} b_{k}^{\dagger} b_{l}=\delta_{i k} \delta_{j l}\left(1-\delta_{i j}\right) c_{i}^{\dagger} c_{j}+\delta_{i j} \delta_{k l} c_{i}^{\dagger} c_{i} c_{k}^{\dagger} c_{k} .
$$

Similar relation can be established for any value of $n$ for the operator linear in $f_{j}^{\dagger} f_{i}$ and of the order $n$ in $b_{j}^{\dagger} b_{i}$. We will not, however, present it explicitly here. Another set of useful relations is:

$$
\begin{aligned}
f_{i}^{\dagger} f_{k}^{\dagger} f_{l} f_{j} & =\left[\delta_{i j} \delta_{k l}-\delta_{i l} \delta_{k j}\right] c_{i}^{\dagger} c_{k}^{\dagger} c_{k} c_{i}, \\
b_{i}^{\dagger} b_{k}^{\dagger} b_{l} b_{j} & =n^{2}\left[\delta_{i j} \delta_{k l}+\delta_{i l} \delta_{k j}\right] c_{i}^{\dagger} c_{k}^{\dagger} c_{k} c_{i} \\
& +n(n-1) \delta_{i l} \delta_{i j} \delta_{i k} c_{i}^{\dagger} c_{i} .
\end{aligned}
$$

As long as the low energy properties are concerned in the low density limit, the introduced truncation of the phase-space is accurate. Similar rules can be formulated for the case of the $\mathrm{FB}_{n}$ complexes. In the case of $n>1$ the derivation of eq. (6) proceeds as follows. After substituting expressions A4 A5 into eqs.45 and, then, into eq.(2), we arrive at the multiple lattice sum of the correlators in eq.(2):

$$
\begin{aligned}
I_{n+1}(\mathbf{Q}) \sim & \int d \mathbf{q}_{2} \ldots d \mathbf{q}_{n+1} \sum_{i_{1} \ldots i_{n+1}, j_{1} \ldots j_{n+1}} \mathrm{e}^{i\left[\left(\mathbf{Q}-\mathbf{q}_{2} \ldots-\mathbf{q}_{n+1}\right) \mathbf{x}_{i_{1} j_{1}}+\mathbf{q}_{2} \mathbf{x}_{i_{2} j_{2}}+\ldots+\mathbf{q}_{n+1} \mathbf{x}_{i_{n+1} j_{n+1}}\right]} \\
& \left\langle\Psi\left|\left(f_{i_{1}}^{\dagger} f_{j_{1}}-\left\langle\Psi\left|f_{i_{1}}^{\dagger} f_{j_{1}}\right| \Psi\right\rangle\right):\left(b_{i_{2}}^{\dagger} b_{j_{2}}-\left\langle\Psi\left|b_{i_{2}}^{\dagger} b_{j_{2}}\right| \Psi\right\rangle\right) \ldots\left(b_{i_{n+1}}^{\dagger} b_{j_{n+1}}-\left\langle\Psi\left|b_{i_{n+1}}^{\dagger} b_{j_{n+1}}\right| \Psi\right\rangle\right):\right| \Psi\right\rangle
\end{aligned}
$$

It is enough to use the normal ordering : ... : for bosonic operators only because just two fermionic operators are involved. The terms with $i_{1} \neq j_{1}$ which project into the one-particle density matrix of the composite fermions contain $2 n$ bosonic operators together with the fermionic pair under $\langle\Psi|\ldots| \Psi\rangle$. These are $\left\langle\Psi\left|f_{i_{1}}^{\dagger} f_{j_{1}}: b_{i_{2}}^{\dagger} b_{j_{2}} \ldots b_{i_{n+1}}^{\dagger} b_{j_{n+1}}:\right| \Psi\right\rangle=$ $\left\langle\Psi\left|f_{i_{1}}^{\dagger}\left(b_{i_{1}}^{\dagger}\right)^{n} f_{j_{1}}\left(b_{j_{1}}\right)^{n}\right| \Psi\right\rangle \delta_{i_{1} i_{2}} \ldots \delta_{i_{1} i_{n+1}} \delta_{j_{1} j_{2}} \ldots \delta_{j_{1} j_{n+1}}=$ $n !\left\langle\Psi\left|c_{i_{1}}^{\dagger} c_{j_{1}}\right| \Psi\right\rangle \delta_{i_{1} i_{2}} \ldots \delta_{i_{1} i_{n+1}} \delta_{j_{1} j_{2}} \ldots \delta_{j_{1} j_{n+1}}$, where the definition (B1) is utilized. This generates the first term in the brackets of eq. (6). The terms with $i_{1}=j_{1}$, after being summed, produce zero due to the conservation of the total number of fermions. This explains the presence of the last term in the brackets of eq.(6). Other terms carry less than $2 n$ bosonic operators averaged together with the fermionic ones. For example, one term which has $2(n-1)$ bosonic operators is $\delta_{i_{2} j_{2}}\left\langle\Psi\left|b_{i_{2}}^{\dagger} b_{i_{2}}\right| \Psi\right\rangle\langle\Psi|\left(f_{i_{1}}^{\dagger} f_{j_{1}}-\left\langle\Psi\left|f_{i_{1}}^{\dagger} f_{j_{1}}\right| \Psi\right\rangle\right)$ : $b_{i_{3}}^{\dagger} b_{j_{3}} \ldots b_{i_{n+1}}^{\dagger} b_{j_{n+1}}:|\Psi\rangle$, where the factor $\delta_{i_{2} j_{2}}$ comes from the relation (B3). It does not contribute to the terms with $i_{1} \neq j_{1}$ because the integration over $\mathbf{q}_{2}$ in eqs. (2 B8) has a structure $\int d \mathbf{q}_{2} \exp \left(i\left(\mathbf{Q}-\mathbf{q}_{2}\right) \mathbf{x}_{i_{1} j_{1}}+i \mathbf{q}_{2} \mathbf{x}_{i_{2} j_{2}}\right) \delta_{i_{2} j_{2}}$ and, thus, selects only terms with $i_{1}=j_{1}$. The same logic applies to other terms with fewer number of bosonic operators. 
[1] T. Stöferle, H. Moritz, K. Günter, M. Köhl and T. Esslinger, Phys. Rev. Lett. 96, 030401 (2006).

[2] G. Thalhammer, K. Winkler, F. Lang, S. Schmid, R. Grimm and J. Hecker Denschlag, Phys. Rev. Lett. 96, 050402 (2006).

[3] C. Ospelkaus, S. Ospelkaus, L. Humbert, P. Ernst, K. Sengstock and K. Bongs, cond-mat/0607581

[4] T. Volz, N. Syassen, D. M. Bauer, E. Hansis, S. Dürr and G. Rempe, cond-mat/0605184

[5] M. Yu. Kagan and I. V. Brodsky, D. V. Efremov, A. V. Klaptsov, Phys. Rev A 70, 023607 (2004).

[6] D. V. Efremov and L. Viverit, Phys. Rev. B 65, 134519(2002);

[7] A. B. Kuklov and B. V. Svistunov, Phys. Rev. Lett. 90, 100401 (2003)

[8] M. Yu. Kagan and D. V. Efremov, Phys. Rev. B 65, 195103 (2002)

[9] E. Mueller, private communication.

[10] M. Lewenstein, L. Santos, M. A. Baranov, and H. Fehrmann, Phys. Rev. Lett. 92, 050401 (2004).

[11] A.F. Andreev, Sov. Phys.- JETP, 41, 1170(1976).

[12] K. Winkler, G. Thalhammer, F. Lang, R. Grimm, J. Hecker Denschlag, and A. J. Daley, A. Kantian, H. P. Büchler, P. Zoller, Nature 441, 853 (2006).

[13] K. Günter, T. Stöferle, H. Moritz, M. Köhl and T. Esslinger, Phys. Rev. Lett. 96, 180402 (2006).

[14] S. Ospelkaus, C. Ospelkaus, O. Wille, M. Succo, P. Ernst, K. Sengstock and K. Bongs, Phys. Rev. Lett. 96, 180403 (2006).

[15] R. Hanbury Brown, R. Q. Twiss, Nature 178, 1046 (1956)

[16] M. Yasuda and F. Shimizu, Phys. Rev. Lett. 77, 3090 (1996).

[17] E. Altman, E. Demler and M.D. Lukin, Phys. Rev. A 70, 013603 (2004).

[18] S.Fölling, F. Gerbier, A. Widera, O. Mandel, T. Gericke and I. Bloch, Nature 434, 481 (2005).

[19] I. B. Spielman, W.D. Phillips and J.V. Porto, cond-mat/0606216
[20] M. Schellekens, R. Hoppeler, A. Perrin, J. Viana Gomes, D. Boiron, A. Aspect, C.I. Westbrook, Science 310, 585 (2005); A. Öttl, S. Ritter, M. Köhl, T. Esslinger, Phys. Rev. Lett. 95, 090404 (2005); J. Esteve, J.-B. Trebbia, T. Schumm, A. Aspect, C.I. Westbrook, I. Bouchoule, Phys. Rev. Lett. 96, 130403 (2006).

[21] M. Greiner, C. Regal and D. Jin, Phys. Rev. Lett. 94, 110401 (2005).

[22] S. Fölling, A. Widera, T. Müller, F. Gerbier, T. Best, T. Rom, D. van Oosten, U. Schneider, I. Bloch, ICAP2006, Insbruck, July 16-22; D. van Oosten, T. Best, T. Rom, U. Schneider, and I. Bloch, Laser Physics 2006, Lausanne, July 24-28.

[23] The composites $\mathrm{FB}_{n}$ can be prepared from the bosonic Mott Insulator state with the integer $k>n$ number of bosons per site. Then, each fermion expels $n$ bosons from a site (which are removed from the system), so that remaining number of bosons is $N_{B}=k N-n N_{F}$.

[24] S. Powell, S. Sachdev and H. P. Buchler Phys. Rev. B 72, 024534 (2005).

[25] M. Greiner, I. Bloch, O. Mandel, T.W. Hänsch and T. Esslinger, Phys. Rev. Lett. 87, 160405 (2001).

[26] M. Köhl, H. Moritz, T. Stöferle, K. Günter and T. Esslinger, Phys. Rev. Lett. 94, 080403 (2004).

[27] In the normal state, the ensembles are mixed states and the fluctuations of the densities exist in each particular realization. This leads to the Hanbury Brown and Twiss effect caused by thermal fluctuations. Instrumental factors, however, can reduce this effect quite significantly. In the experiment 18] no Hanbury Brown and Twiss-type Bragg structure was observed in the thermal cloud. This was attributed to diminishing the signal due to the cloud size increase and noise from the background [18].

[28] E. M. Lifshitz and L. P. Pitaevskii, Statistical Physics. Part 2. Course of Theoretical Physics. Vol. 9, Pergamon Press, 1980.

[29] G.H. Wannier, Phys.Rev. 52, 191(1937). 